\title{
Dielectric properties and Electrical Conductivity Studies on Neodymium and Chromium co-doped Gadolinium Scandium Gallium Garnet (GSGG:Nd:Cr)
}

\author{
A Rama Krishna ${ }^{1 *}$, G Sathaiah ${ }^{2}$ and Lalitha Sirdeshmukh ${ }^{3}$ \\ ${ }^{I}$ Department of Physics, Malla Reddy College of Engineering, Maisammaguda(Vi), Dhulapally(M), \\ Secunderabad - 500 014, India. \\ ${ }^{2 \notin 3}$ Department of Physics, Kakatiya University, Warangal-506 009, India.
}

\begin{abstract}
The dielectric properties (Dielectric constant ' $\varepsilon$ ' and Dielectric loss 'Tan $\delta$ ') of a Neodymium and Chromium co-doped Gadolinium Scandium Gallium Garnet $\left(\mathrm{Gd}_{3} \mathrm{Sc}_{2} \mathrm{Ga}_{3} \mathrm{O}_{12}: \mathrm{Nd}^{3+}: \mathrm{Cr}^{3+}\right)$ single crystal were measured in the frequency range from $1 \mathrm{kHz}$ to $1 \mathrm{MHz}$ and in the temperature range from room temperature to $700^{\circ} \mathrm{C}$. Electrical conductivity $(\sigma)$ was calculated using the data on $\varepsilon$ and tan $\delta . D C$ conductivity was also measured in the same temperature range. The electrical conductivity in this garnet is discussed in light of existing data.
\end{abstract}

Keywords: Dielectric constant, Dielectric loss, ac conductivity, garnet

\section{Introduction}

Rare earth garnets find immense applications in magnetic bubble memory devices [1], laser hosts [2] and in radio-electronics and microwave devices [3]. Some of the applications of non-iron garnets such as Gadolinium Gallium Garnet $\left(\mathrm{Gd}_{3} \mathrm{Ga}_{5} \mathrm{O}_{12}\right)$ and Yttrium Aluminium Garnet $\left(\mathrm{Y}_{3} \mathrm{Al}_{5} \mathrm{O}_{12}\right)$ are their use in quantum electronics and microelectronics [4]. GGG is used as substrate material for devices. Rare earth doped gallium and aluminium garnets are excellent hosts for lasers [5,6]. Because of their technical importance several attempts have been made to grow these materials and study their physical properties [7].

The optical, magnetic and other physical properties of rare earth garnets have been extensively studied by several earlier workers, but the study on the dielectric properties and electrical conduction are meagre. Literature survey shows that the work on electrical properties on garnets is limited. Electrical conductivity in GGG was reported by Krishan Lal and Jhans [8]. Dielectric constants on several gallium garnets at room temperature were reported by Shannon et. al. [9]. The electrical transport mechanism in Dysprosium Iron Garnet (DyIG) was studied by Vijayee Ram Yadav and H. B. Lal [10]. The dielectric properties of some gallium garnets, aluminium and iron garnets were studied by the authors $[11,18,19]$. There was a study on the spectroscopic, optical, and thermo mechanical properties of GSGG:Nd:Cr [12], but there was no study of the dielectric properties on this garnet. In the present paper the authors report their systematic study of the dielectric constant $(\varepsilon)$ and dielectric loss $(\tan \delta)$ as a function of frequency and temperature. The dc conductivity of the garnet has been measured with temperature. Results are discussed.

\section{Experimental}

A highly polished single crystal of $\mathrm{Gd}_{3} \mathrm{Sc}_{2} \mathrm{Ga}_{3} \mathrm{O}_{12}$ doped with $5 \%$ of Neodymium and $0.3 \%$ of Chromium in rectangular shape with $0.72 \mathrm{~cm}^{2}$ surface areas and $0.19 \mathrm{~cm}$ thickness is used in the present investigations. The crystal is greenish in colour. Measurements were made perpendicular to (111) direction. The crystal is coated with silver paint for good electrical contact with the electrodes and to remove air gaps.

The dielectric constant $(\varepsilon)$ and loss $(\tan \delta)$ were measured using a GR-1620A capacitance measuring assembly, in conjunction with a locally built three terminal cell. The measurements were carried out in the frequency range from $100 \mathrm{~Hz}$ to $100 \mathrm{kHz}$ and in the temperature range from room temperature to $700^{\circ} \mathrm{C}$. High temperature measurements were made in a furnace with a programmable temperature controller (RKC, REX P90) with accuracy in temperature of $\pm 1^{\circ}$.

The $\mathrm{AC}$ conductivity is calculated using the data on $\varepsilon$ and $\tan \delta$ by:

$$
\sigma_{a c}=\epsilon \epsilon_{o} \omega \tan \delta
$$

The DC conductivity measurements were done using a KEITHLEY 610C electrometer amplifier in conjunction with a locally built sample holder.

\section{Results}

Initially the crystal was annealed at $800^{\circ} \mathrm{C}$, in air for several hours before taking the measurements. The variation of dielectric constant $(\varepsilon)$ and dielectric loss $(\tan \delta)$ as a function of frequency at room temperature as 
shown in figures 1 and 2. A little decrease in dielectric constant and tan $\delta$ are observed with frequency. The room temperature dielectric constant and loss values are found to be 12 and $3 \times 10^{-3}$ respectively at $100 \mathrm{kHz}$ frequency. Annealing effects were seen as the measurements were repeated after annealing the sample a few times.

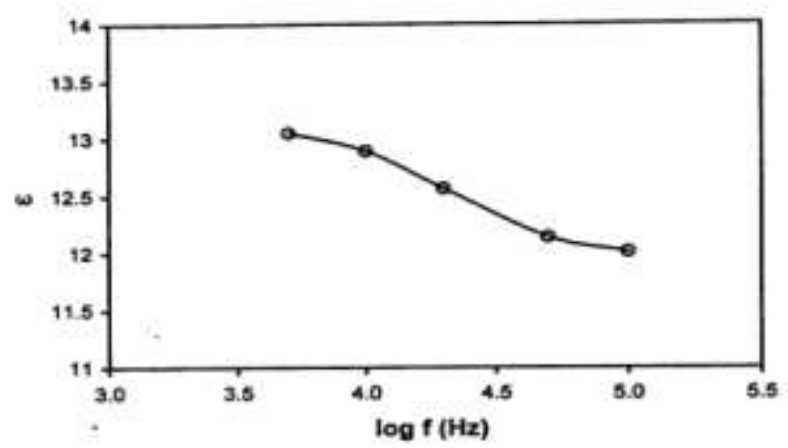

Figure 1. Variation of $\varepsilon$ with frequency for GSGG:Nd:Cr

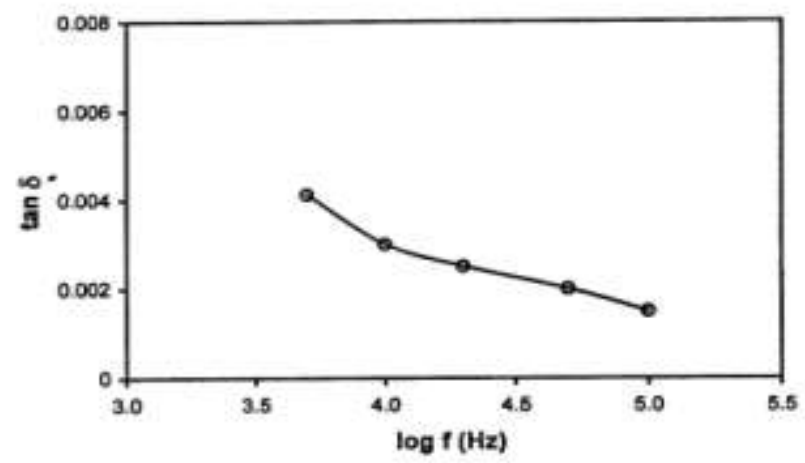

Figure 2. Variation of $\tan \delta$ with frequency for GSGG:Nd:Cr

The dielectric constant variation as a function of temperature for different frequencies is shown in figure 3. The dielectric constant increases slowly and is almost independent of frequency up to $450^{\circ} \mathrm{C}$ after which it increases rapidly with temperature which is frequency dependent. A peak is observed at $560^{\circ} \mathrm{C}$ for all frequencies with higher values of $\varepsilon$ for lower frequencies. It may be noted that the peaks are sharp and appear at the same temperature. Beyond $600^{\circ} \mathrm{C}$ a large increase in dielectric constant is again observed for all frequencies.

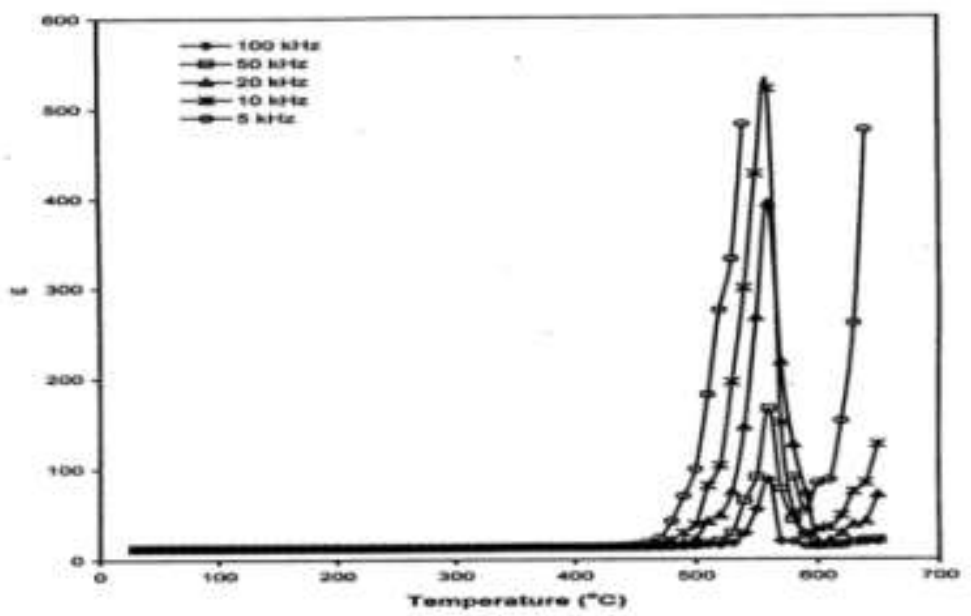

Figure 3. Variation of dielectric constant with temperature for GSGG:Nd:Cr 
The temperature dependent variation of dielectric loss $(\tan \delta)$ for different frequencies is shown in figure 4 . It can be seen that $\tan \delta$ varies slowly in a usual manner up to $430^{\circ} \mathrm{C}$, beyond which the increase is rapid. A peak for all frequencies centered at $560^{\circ} \mathrm{C}$ is observed in the loss curves similar to those observed in the curves of dielectric constant versus temperature. Beyond $600^{\circ} \mathrm{C}$, again a rapid, frequency dependent increase in $\tan \delta$ is observed.

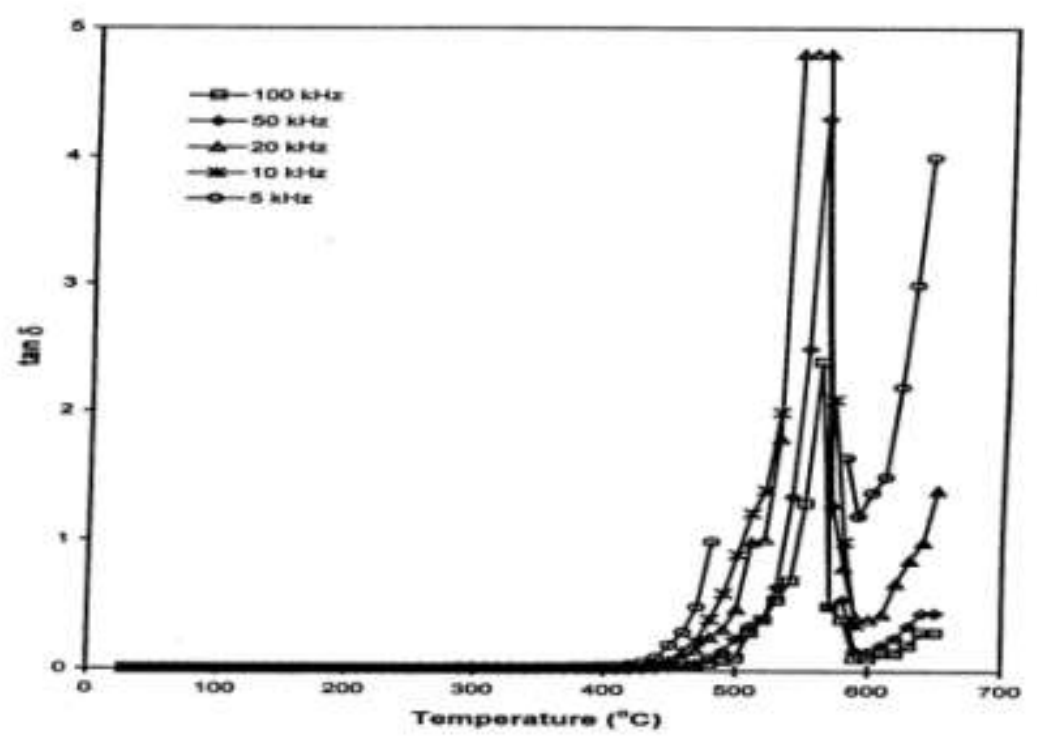

Figure 4. Variation of dielectric loss with temperature for GSGG:Nd:Cr

The AC conductivity variation with reciprocal temperature for the sample for different frequencies is shown as Arrhenius plots in figure 5. A frequency dependent variation is seen from room temperature to $400^{\circ} \mathrm{C}$ which is independent of temperature. From $400^{\circ} \mathrm{C}$ to $650^{\circ} \mathrm{C} \mathrm{AC}$ conductivity increases which is frequency independent.

Figure 6 shows the variation of DC conductivity with reciprocal temperature. An increase is observed beyond $400^{\circ} \mathrm{C}$ temperature. DC conductivity is found to be lower than AC conductivity.

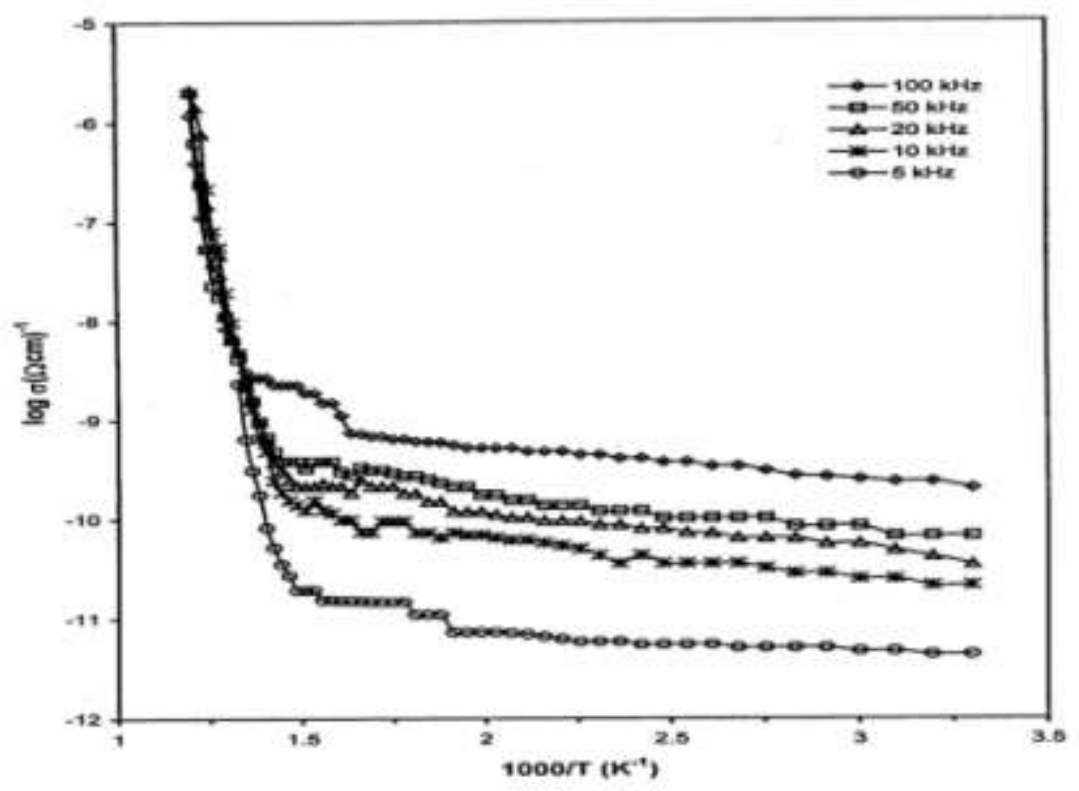

Figure 5. Variation of AC conductivity with reciprocal temperature for GSGG:Nd:Cr 


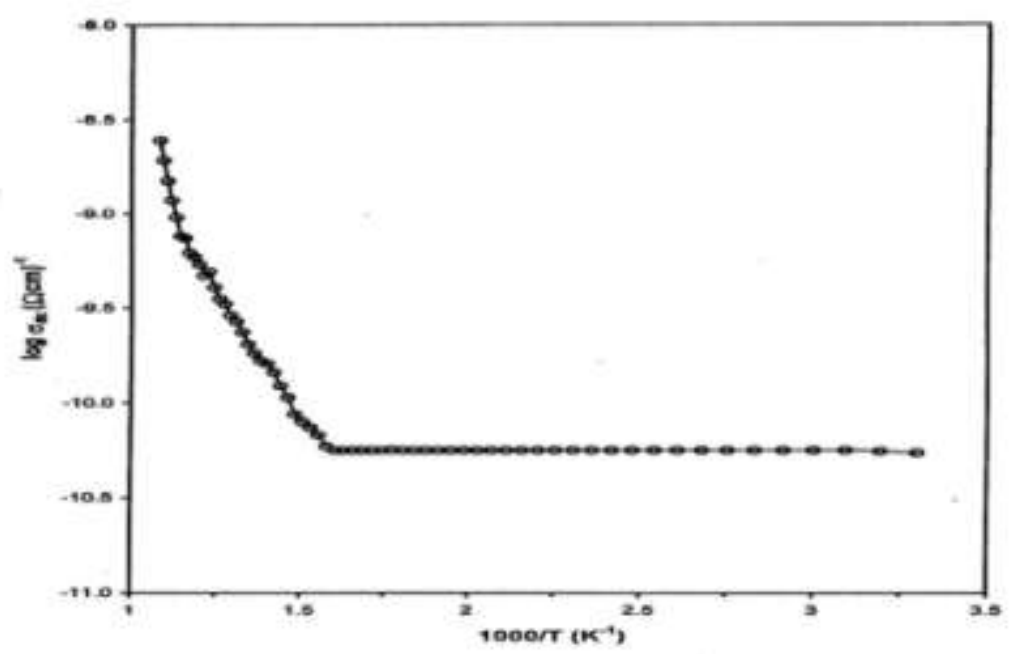

Figure 6. Variation of DC conductivity with reciprocal temperature for GSGG:Nd:Cr

\section{Discussion}

The variation of dielectric constant and dielectric loss with frequency at room temperature shows the usual behaviour as observed for the ionic crystals [13] and other garnet samples, viz., pure and $\mathrm{Nd}$ doped Gadolinium Gallium Garnets (GGG) [11], Neodymium Gallium Garnet (NdGG) [14], pure and Nd doped YAG [18]. The variation for the two samples, both pure and $\mathrm{Nd}$ doped one shows distinct difference, which is more pronounced for the variation of loss with temperature. Studies on luminescence by Andrichuk et. al. [15] have indicated that the intensity of luminescence emitted by GGG depends on the structural disorder. The fine structure absorption spectra (EXAFS) studied by Jun Dong and Kunquan Lu [16] has indicated the site exchange between $\mathrm{Ga}$ and $\mathrm{Gd}$ atoms in $\mathrm{Gd}_{3} \mathrm{Ga}_{5} \mathrm{O}_{12}$ and small displacements of atomic positions results in noncubic symmetry of garnets. The non-cubic symmetry is described by R $\overline{3}$. It is a small distortion from Ia $\overline{3} \mathrm{~d}$ space group. In the case of Nd doped Yttrium aluminium garnet (YAG:Nd), Bagdasarov et. al. [17] have shown that the change in the structure is brought either by light pumping with UV illumination or temperature. They have observed anomalies in dielectric constant $(\varepsilon)$, dielectric loss $(\tan \delta)$ and conductivity around $770 \mathrm{~K}$. These anomalies observed around $450^{\circ} \mathrm{C}$ and $630^{\circ} \mathrm{C}$ could be understood by comparing with the report on Nd doped YAG [17]. In case of YAG the present authors also observed [18] promising peaks in the dielectric constant $(\varepsilon)$ and dielectric loss $(\tan \delta)$ around $500^{\circ} \mathrm{C}$ for all frequencies. This is attributed to a possible structural phase transition. In the present case also a similar type of changes are expected due to the presence of $\mathrm{Nd}$ in the ' $\mathrm{c}$ ' sub lattice.

The AC conductivity variation versus reciprocal temperature shows similar variation for both the samples. Up to $400^{\circ} \mathrm{C}$ the conductivity is temperature independent and beyond this temperature it is frequency independent with a sharp increase with temperature.

Referring to the behaviour in the case of iron garnets studied by the present authors [19], the possible mechanism of conduction is by hopping of electrons. This hopping conduction is due to the exchange of electrons between the divalent $\mathrm{Fe}^{2+}$ and the trivalent $\mathrm{Fe}^{3+}$ ions. But in the case of non-magnetic garnets such as GGG and GSGG the exchange of electrons is not possible. Hence the possible mechanism of conduction may be due to the motion of ions. The low value of conductivity at room temperature, which is of the order of $10^{-12} \Omega^{-}$ ${ }^{1} \mathrm{~cm}^{-1}$, also indicates the absence of electronic conduction. The probable charge carriers are the oxygen ions.

\section{Conclusions}

The dielectric constant $(\varepsilon)$ and dielectric loss $(\tan \delta)$ for an Nd, Cr co-doped GSGG garnet are found. The variation with temperature is similar to those of other Nd doped garnet crystals. Peaks in $\varepsilon$ and $\tan \delta$ are observed at $560^{\circ} \mathrm{C}$. A distinct difference from the earlier observations is that the peaks observed are very sharp. There is also an indication of second set of peaks in $\varepsilon$ and $\tan \delta$ at higher temperatures, which require further confirmation by investigation. The high value of conductivity (around $10^{-6} \Omega^{-1} \mathrm{~cm}^{-1}$ ) could be due to the presence of transition metal ion $\mathrm{Cr}^{3+}$ in the sample. The conductivity is much higher than other samples studied earlier. The cause for these observations is due to the presence of an electric dipole formed by the oxygen defect and $\mathrm{Nd}^{3+}$ ion. 


\section{Acknowledgements}

The authors thank the 'Solid State Laboratories, New Delhi for the gift of the garnet sample. The lab facilities provided by the Materials Research Lab, Osmania University, Hyderabad are greatly acknowledged.

\section{References}

[1] U. F. Gianola, D. H. Smith, A. A. Thieie and L. G. Van Uitert, IEEE Trans. Magn. MAG., 5, 1969, p 558

[2] J. E. Geusic, H. M. Marcos and L. G. Van Uitert, Appl. Phys. Lett., 4, 1964, p 182

[3] J. F. Dillon Jr, J. Magn. Magn. Mater. (Netherlands), 84, 1990, p213

[4] S. Krupicka, The Physics of ferrites and magnetic oxides related to them (Russian Translation) Mir Publishers, $1^{\text {st }}$ ed, 1976

[5] F. Johnson and K. A. Ingersoll, J. Appl. Phys., 44, 1973, p 5444

[6] W. T. Silfvast, 1998 Laser Fundamentals, Cambridge University Press, (South Asian paperback edition)

[7] C. P. Khattak and F. F. Y. Wang, Hand Book on the Pysics and Chemistry of Rare Earths, ed K A Gaschneidner Jr and L Eyring North Holland Publishing Company, New York, Chap. 29, 1979, pp 525-601

[8] Krishan Lal and H. K. Jhans, J. Phys. C. Solid State Physics, 10, 1977, p 1315

[9] R. D. Shannon, M. A. Subramanian, T. H. Allik, M. R. Kokta, M. H. Randles and G. R. Rossman, J. Appl. Phys., 67, 1990, p 3798

[10] Vijayee Ram Yadav and H. B. Lal, Jap. J. Appl. Phys. , 18, 1979, pp 2229-2232

[11] A. Rama Krishna, Dielectric properties and Electrical conductivity studies on some Rare earth Garnets, doctoral dissertation, Kakatiya University, Warangal, India, 2003

[12] W. F. Krupke, M. D. Shinn, J. E. Marion, J. A. Caird and S. E. Stokowski, JOSA B, 3(1), 1986, pp 102-114

[13] Lalitha Sirdeshmukh, G. Sathaiah and Prameela Devi, Phys. Stat. Sol. (a), 99, 1987, p 631

[14] A. Rama Krishna, K. Krishna Kumar, G. Sathaiah and Lalitha Sirdeshmukh, presented at the $5^{\text {th }}$ IUMRS-ICA, International conference in Asia, Bangalore, 1998 (unpublished)

[15] V. A. Andrichuk, L. G. Volzhenakaya, Y. M. Zakharko and Yu. V. Zorenko, Sov. Phys. - Solid State (USA), 29, 1987, p 131

[16] Jun Dong and Kunquan Lu, Phys. Rev. B, 43, 1991, p 8808

[17] Kh. S. Bagdasarov, N. Ya. Bagdanov, V. I. Kalinin, E. I. Kut'in and E. M. Uyukin, Crystallogra. Rep., 38, 1993 , p 361

[18] A. Mallikarun Reddy, A. Rama Krishna, G. Sathaiah and Lalitha Sirdeshmukh, Condensed Matter Physics, Narosa Publishing House, New Delhi, 1999, pp 215-219

[19] Lalitha Sirdeshmukh, K. Krishna Kumar, S. Bal Laxman, A. Rama Krishna and G. Sathaiah, Bull. Mater. Sci., 21(3), 1998, pp 219226 